\title{
Letter
}

\section{Cosmological imprints of string axions in plateau}

\author{
Jiro Soda ${ }^{1, a}$, Yuko Urakawa ${ }^{2,3, b}$ \\ ${ }^{1}$ Department of Physics, Kobe university, Kobe 657-8501, Japan \\ 2 Department of Physics and Astrophysics, Nagoya University, Chikusa, Nagoya 464-8602, Japan \\ ${ }^{3}$ Institut de Ciencies del Cosmos, Universitat de Barcelona, Marti i Franques 1, 08028 Barcelona, Spain
}

Received: 6 August 2018 / Accepted: 13 September 2018 / Published online: 26 September 2018

(C) The Author(s) 2018

\begin{abstract}
We initiate a study on various cosmological imprints of string axions whose scalar potentials have plateau regions. In such cases, we show that a delayed onset of oscillation rather generically leads to a parametric resonance instability. In particular, for ultralight axions, the parametric resonance can enhance the power spectrum slightly below the Jeans scale, alleviating the tension with the Lyman $\alpha$ forest observations. We also argue that a sustainable resonance can lead to an emission of gravitational waves at the frequency bands which are detectable by gravitational wave interferometers and pulsar timing arrays and also to a succeeding oscillon formation.
\end{abstract}

\section{Introduction}

Compactifications in string theory generically predict various axions in 4D low energy effective field theory. Exploring imprints of axions in cosmology provides an important tool to probe extra dimensions predicted in string theory [1]. Phenomenological impacts of axions have been mostly studied by considering the quadratic potential. However, once an axion is away from the potential minimum, the potential deviates from the quadratic form. In particular, when the dilute instanton gas approximation does not hold, the scalar potential can be more flatten than the conventional cosine form $[2,3]$. Therefore, it is worth investigating phenomenological consequences of axions with such plateau regions in their scalar potentials.

Distinctively, the axions which were located at such plateau regions generically undergo a parametric resonance after their onsets of oscillations, which exponentially enhances the modes in the resonance bands and potentially leaves various phenomenological impacts. This parametric resonance instability has been widely studied in the context

\footnotetext{
a e-mail: jiro@phys.sci.kobe-u.ac.jp

be-mail: urakawa.yuko@h.mbox.nagoya-u.ac.jp
}

of reheating after inflation. (For reheating, see e.g., historical papers [4-6] and [7].) In Refs. [8-12], it was shown that when the potential is shallower than the quadratic form, the instability leads to a fragmented configuration of the oscillating scalar field, the so called oscillon (see also Refs. [13,14]). In Ref. [15], it was shown that the oscillating axion can induce resonance phenomena also in gravitational waves.

In string theory, there appear axions in a wide mass range. For example, the large volume scenario predicts the presence of extremely light axions (see e.g., Refs.[16,17]). The onset time of the oscillation varies, depending on the mass scale of the axion. In particular, the ultra-light axion (ULA) whose mass is of $O\left(10^{-22} \mathrm{eV}\right)$ commences the oscillation before the matter-radiation equality and behaves as a fuzzy dark matter. The ULA has been often discussed in the context of the small scale issues of $\Lambda \mathrm{CDM}[18,19]$. Meanwhile, it was argued that the ULA with $m \simeq 10^{-22} \mathrm{eV}$ is marginally incompatible with the Lyman $\alpha$ forest observations, since the ULA smooths out the small scale structures too much [20]. It is interesting to see whether the parametric resonance instability can relax the tension with the Lynman $\alpha$ forest observations or not.

\section{Setup of problem}

In order to study dynamics of string axions whose potentials have a shallower region than the quadratic potential, we consider a canonical scalar field $\phi$ with a scalar potential $V(\phi)$ given by $V(\phi)=(m f)^{2} \tilde{V}(\tilde{\phi})$ with $\tilde{\phi} \equiv \phi / f$. Here, $\tilde{V}(\tilde{\phi})$ satisfies the following properties: i) $\tilde{V}(\tilde{\phi}) \rightarrow \tilde{\phi}^{2} / 2$ in the limit $\tilde{\phi} \rightarrow 0$, ii) $\tilde{V}(\phi) / \tilde{\phi}^{2} \rightarrow 0$ in the limit $|\tilde{\phi}| \rightarrow \infty$. Since the axion is a pseudo-scalar, it is reasonable to impose additionally $Z_{2}$ symmetry on the potential. The parameter $f$ agrees with the decay constant in the case with the cosine potential. Roughly speaking, $m$ determines the onset time of the oscillation (under a certain initial condition) and $f$ determines the energy density for a given $m$. 
In this paper, we will investigate phenomenological consequences of the axions with a potential which satisfies the conditions (i) and (ii). Considering a situation where the selfinteraction is much more important than the gravitational interaction, we solve the Klein-Gordon (KG) equation

$\square \phi-V_{\phi}=0$

in a fixed geometry.

\section{Evolution of the homogeneous mode}

First, we consider the evolution of the homogeneous mode of $\phi$. In the following, we assume the background expansion law as $a \propto t^{p}$ with $p>0{ }^{1}$. When the field $\phi$ does not dominate the universe, the power $p$ cannot be determined only from the dynamics of $\phi$. Then, the KG equation for the homogeneous mode is given by

$\frac{d^{2} \tilde{\phi}}{d x^{2}}+\frac{3 p}{x} \frac{d \tilde{\phi}}{d x}+p^{2} \frac{d \tilde{V}}{d \tilde{\phi}}=0$

with $x \equiv m / H=m t / p$. Notice that all the dimensionful parameters dropped out from the equation and the onset time of oscillation, $x_{o s c}$, is solely determined by the initial conditions $\tilde{\phi}_{i} \equiv \tilde{\phi}\left(x_{i}\right)$ and $\tilde{\phi}_{x, i} \equiv d \tilde{\phi} / d x\left(x_{i}\right)$. The Hubble parameter at $x_{o s c}$ for a given $m$ is determined as $H_{o s c}=m / x_{o s c}$. When the axion stays at a plateau, it behaves as a cosmological constant. In fact, when the potential gradient term is negligible, the equation of motion (2) can be solved analytically as $\tilde{\phi}(x)=C_{1}+C_{2} x^{1-3 p}$. Meanwhile, around the bottom of the potential with $|\tilde{\phi}| \ll 1$, Eq. (2) can be also solved analytically. However, in general, Eq. (2) can be solved only numerically in the intermediate range.

Notice that when $\phi$ is located at the plateau region at the onset of oscillation, the oscillation does not necessarily take place around $x \simeq 1$ or $H \simeq m$. What will be discussed in this paper applies rather generically, in case the scalar potential satisfies the conditions (i) and (ii). However, for a concrete analysis, as an example, we consider an $\alpha$ attractor type potential, given by

$\tilde{V}(\tilde{\phi})=\left[1+c(\tanh \tilde{\phi})^{2}\right]^{-1} \times(\tanh \tilde{\phi})^{2} / 2$

with $c \geq 0$. The $\alpha$ attractor model was considered as a generalization of the superconformal models [21-23]. This potential is shown in Fig. 1 for different values of $c$. For

\footnotetext{
${ }^{1}$ When the axion dominates the universe, we need to determine the expansion law consistently. However, this point is not very important in discussing the dynamics of inhomogeneous modes, especially when the onset of the oscillation is delayed.
}

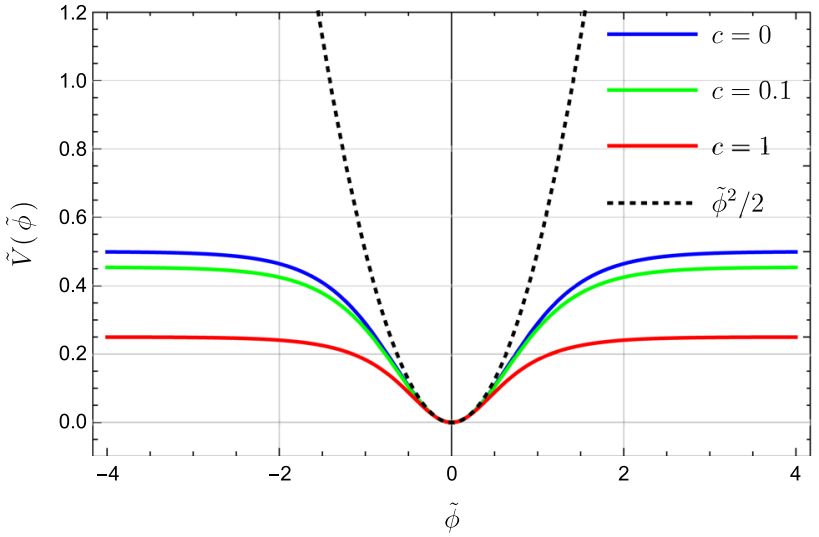

Fig. 1 The plot shows the potential shape of the $\alpha$ attractor type potential (3) for different values of $c$

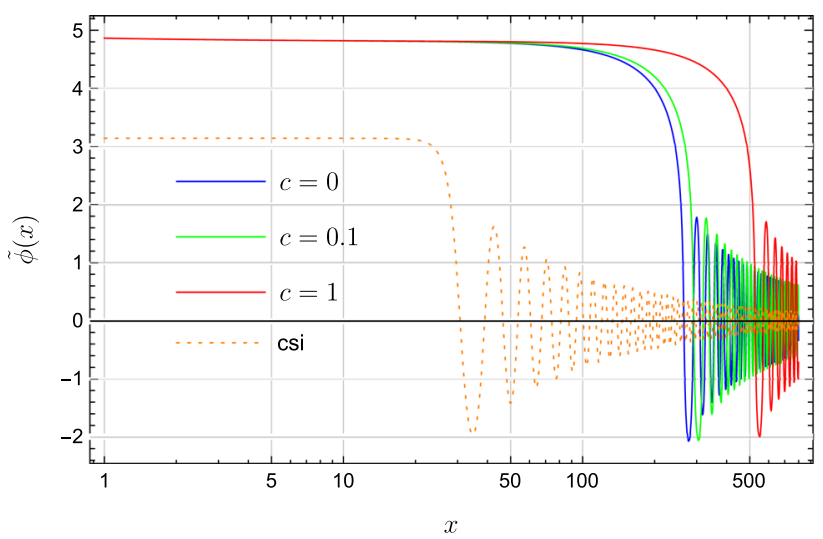

Fig. 2 This plot shows the time evolution of $\tilde{\phi}$ in RD

$|\tilde{\phi}|<1$, the second derivative of the potential is given by $\tilde{V}_{\tilde{\phi} \tilde{\phi}}=1-2(2+3 c) \tilde{\phi}^{2}+O\left(\tilde{\phi}^{4}\right)$, i.e., the curvature of the potential becomes smaller for a larger value. Figure 2 shows that the oscillation starts at $x_{o s c} \gg 1$, when we choose the initial condition $\tilde{\phi}_{i}=5$ and $\tilde{\phi}_{x, i}=-1$, starting at the plateau region. As was later pointed out in Ref. [24], $x_{\text {osc }}$ can be roughly estimated as $x_{o s c} \sim \sqrt{\left|\tilde{\phi}_{i} / \tilde{V}_{\tilde{\phi}}\left(\tilde{\phi}_{i}\right)\right|}$, indicating that the onset of the oscillation delays, taking $x_{o s c} \gg 1$, when $\phi$ was initially located at a potential region which is much shallower than the quadratic potential. (When the plateau is wide enough, the evolution does not much depend on the initial velocity because of the over damping.) The orange dotted line shows the time evolution for the conventional cosine potential $\tilde{V}(\tilde{\phi})=1-\cos \tilde{\phi}$ with $\tilde{\phi}_{i}=\pi$ and $\tilde{\phi}_{x, i}=-10^{-4}$. Even with this fine-tuned initial condition, the oscillation starts much earlier than the plateau case.

When $\phi$ starts to oscillate before the matter-radiation equality, we can estimate the decay constant by equating the energy density of the radiation $\rho_{\gamma}^{e q}$ with that of dark matter $\rho_{m}^{e q}=\rho_{\phi}^{e q} / \beta_{\phi}$, where $\beta_{\phi}$ denotes the fraction of the ULA among the total dark matter, as 
$f \simeq \frac{\beta_{\phi}^{\frac{1}{2}}}{m}\left(\frac{\rho_{e q}^{\frac{1}{3}} H_{o s c}^{2}}{8 \pi G}\right)^{\frac{3}{8}} \simeq\left(\frac{10^{-22} \mathrm{eV}}{m}\right)^{\frac{1}{4}} \frac{\beta_{\phi}^{\frac{1}{2}}}{x_{o s c}^{\frac{3}{4}}} 10^{17} \mathrm{GeV}$,

where the quantities with the index $e q$ denote those at the equal time and the quantities with the index osc denote those at the onset of the oscillation. Here, taking into account that the kinetic energy and the potential energy are comparable at the onset of the oscillation, we used $\rho_{\phi}^{o s c} \simeq 2 V_{\text {osc }} \simeq(m f)^{2}$. Thus, once $x_{o s c}$ is given by solving Eq. (2), the decay constant $f$ is determined by Eq. (4) for a given $m$.

\section{Parametric resonance instability}

Next, we study the evolution of the inhomogeneous modes. The perturbed KG equation for the axion is given by $\ddot{\varphi}+$ $3 H \dot{\varphi}+(k / a)^{2} \varphi+V_{\phi \phi} \varphi=0$, where $\varphi$ is the perturbed axion field and we neglected the metric perturbations. According to our numerical analysis, the metric perturbation does not play a crucial role at least in the early stage of the resonance instability, where the linear analysis can apply. Again, we can rewrite the perturbed KG equation for $\tilde{\varphi} \equiv \varphi / \varphi_{i}$, which is normalized by the initial value $\varphi_{i}$, in a dimensionless form

$\frac{d^{2} \tilde{\varphi}_{k}}{d x^{2}}+3 \frac{p}{x} \frac{d \tilde{\varphi}_{k}}{d x}+p^{2} \tilde{k}^{2}\left(\frac{x_{i}}{x}\right)^{2 p} \tilde{\varphi}_{k}+p^{2} \tilde{V}_{\tilde{\phi} \tilde{\phi}} \tilde{\varphi}_{k}=0$

where we used $k /(a m)=\tilde{k}\left(x_{i} / x\right)^{p}$ with $\tilde{k} \equiv k /\left(a_{i} m\right)$. Depending on the choice of the initial time, the corresponding wavenumber $\tilde{k}$ varies, while $k / a$ is independent of the choice of the initial time. Here, we choose $x_{i}=1 / 10$.

Figure 3 shows the time evolution of $\tilde{\varphi}_{k}$ for the same potentials as in Fig. 2 during RD. The fluctuation $\tilde{\varphi}_{k}$ for the cosine potential under the same initial condition as in Fig. 2 grows much less than the one for the $\alpha$ attractor type

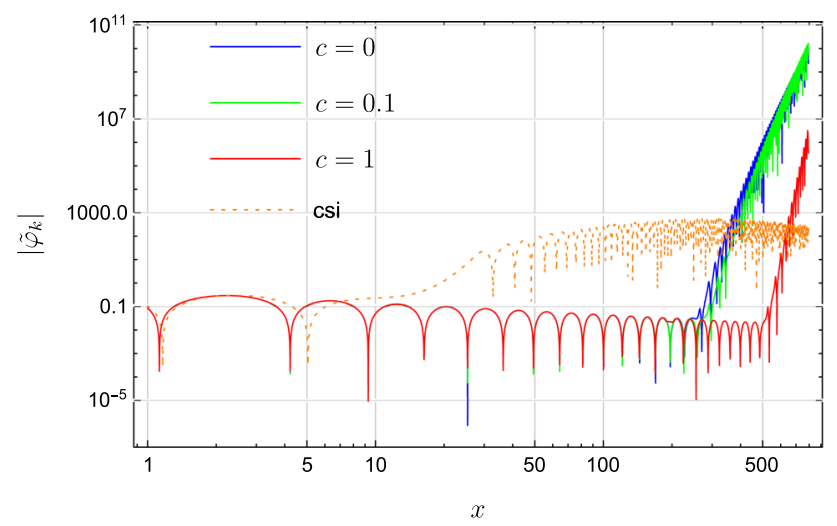

Fig. 3 This plot shows the time evolution of $\tilde{\varphi}_{k}$ with $\tilde{k}=10$ under the initial condition $\tilde{\phi}_{i}=5$ during RD
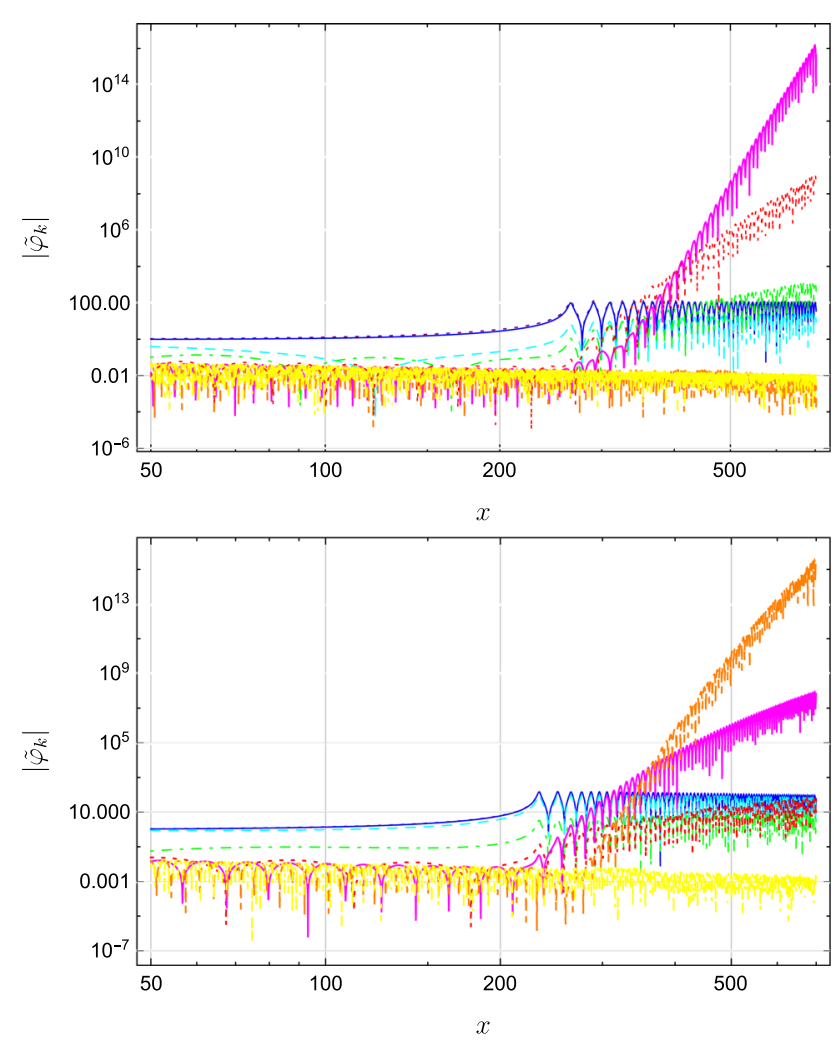

Fig. 4 These plots show the time evolutions of $\tilde{\varphi}_{k}$ during RD (up) and MD (bottom) for different wavenumbers $\tilde{k}: \tilde{k}=10^{-1}$ (purple, dotted), $\tilde{k}=10^{-1 / 2}$ (blue), $\tilde{k}=1$ (light blue, dashed), $\tilde{k}=10^{1 / 2}$ (green, dotdashed), $\tilde{k}=10$ (red, thick-dotted), $\tilde{k}=10^{3 / 2}$ (magenta, thick), $\tilde{k}=$ $10^{2}$ (orange, thick-dashed), and $\tilde{k}=10^{5 / 2}$ (yellow, thick-dotdashed). Here, we choose $c=0$. The parametric resonance instability is slightly more efficient in $\mathrm{RD}$ than in MD, since the comic expansion is slower

potential. Figure 4 shows the time evolution of $\tilde{\varphi}_{k}$ for different wavenumbers $\tilde{k}$ during RD (up) and MD (bottom), when $\tilde{V}$ is given by Eq. (3) with $c=0$. The modes with $\tilde{k}=10^{-1}$ and $\tilde{k}=10^{-1 / 2}$ got slightly enhanced just after the onset of the oscillation due to the tachyonic instability. However, the conventional tachyonic instability is not very efficient, because the second derivative of the potential soon starts to oscillate, taking both positive and negative values. In our accompanying paper [24], where we performed a more detailed analysis, we showed that a different type of resonance instability, dubbed the flapping resonance instability sets in, when the curvature of the potential oscillates between negative and positive values ${ }^{2}$.

In order to understand the instability more intuitively, here let us analyze the equation (5), neglecting the Hubble friction. Around the bottom of the potential, i.e., $\tilde{\phi}<1$, the homogeneous mode of the axion oscillates with the frequency $\left|\tilde{V}_{\tilde{\phi} \tilde{\phi}}\right|^{1 / 2} \simeq 1$ and the solution is given by $\tilde{\phi}=\tilde{\phi}_{*} \cos z$ with

\footnotetext{
${ }^{2}$ For the $\alpha$ attractor potential with larger values of $c$, the flapping resonance tends to be more prominent than the narrow resonance [24].
} 
$z=m t$. Using this solution, Eq. (5) is given by Mathieu equation as

$\frac{d^{2}}{d z^{2}} \tilde{\varphi}+(A-2 q \cos 2 z) \tilde{\varphi}=0$,

where $A$ and $q$ are defined as

$A \equiv\left(\frac{k}{m a_{o s c}}\right)^{2}+1-(2+3 c) \tilde{\phi}_{*}^{2}$

$q \equiv \frac{2+3 c}{2} \tilde{\phi}_{*}^{2}$

Here, $a_{\text {osc }}$ denotes the scale factor at around the onset of the oscillation. The parametric resonance takes place for the narrow band $A \simeq n^{2}$, where $n$ is an integer. The width of the resonance band is proportional to $(q / A)^{n}$. The dominant growing mode is the $n=1$ mode and the growth rate $\gamma$ with $\tilde{\varphi} \propto e^{\gamma x}$ is given by $\gamma \simeq q / 2=(2+3 c) \tilde{\phi}_{*}^{2} / 4$. Notice also that the resonance band becomes wider for a larger $c$ as shown in Fig. 3. The resonance wavenumbers can be predicted from the first resonance band of Mathieu equation, leaving aside factor deviations.

The cosmic expansion makes the parametric resonance instability inefficient mainly due to the following two effects: first, the amplitude $\tilde{\phi}_{*}$ decreases due to the Hubble friction, reducing the growth rate and second, more importantly here, the physical wavenumbers in the resonance band(s) are red shifted. When the gradient of the potential is shallower, the onset of the oscillation gets more delayed, i.e., $x_{o s c} \gg 1$. Then, when the parametric resonance instability sets in, the redshift of the resonant modes due to the cosmic expansion is not effectively important any more. This leads to the sustainable resonant growth without being disturbed by the cosmic expansion. Because of that, an efficient resonance instability requires a shallower potential than the quadratic potential region, where the oscillation takes place at $x_{o s c} \simeq 1$. The exponential growth continues until the time when the rescattering due to the backreaction becomes important, i.e., $|\varphi / f| \simeq O$ (1) (see, e.g., Ref. [7]). As is shown in Fig. 3, for a larger $c$, the resonance instability proceeds more rapidly, since the oscillation starts later. To visualize this aspect more clearly, for a reference, we also plotted the time evolution of $\tilde{\phi}$ and $\tilde{\varphi}$ for the cosine potential in Figs. 2 and 3, respectively.

In Figs. 3 and 4, where we choose the initial field value $\tilde{\phi}_{i}=5$, the parametric resonance persists without being disturbed by the cosmic expansion. On the other hand, when we choose a smaller value of $\left|\tilde{\phi}_{i}\right|$ as an initial condition, the parametric resonance can persist only in a shorter period, leaving only a milder enhancement of the fluctuation.

\section{Jeans scale}

It is known that the ULA has an emergent pressure on small scales and the Jeans wavenumber is given by $k_{J}(a) \simeq$ $\sqrt{m H} a$, where we used $c_{s} \simeq k /(2 m a)[18,19]$. When the ULA dark matter becomes the dominant component of the universe for $a>a_{e q}$, the structures below the Jeans length are smoothed out. As is shown in Fig. 4, the parametric resonance instability takes place for the wavenumbers slightly above the Jeans wavenumber. This can be understood as follows. The resonance wavenumber in the first band $k_{r}$ satisfies $k_{r} /\left(m a_{o s c}\right) \simeq O(1)$. Therefore, using $k_{J} /(m a) \simeq 1 / \sqrt{x}$, we find a universal relation $k_{r} \simeq \sqrt{x_{\text {osc }}} k_{J}\left(a_{e q}\right)$. When the scalar potential of the ULA dark matter has a plateau region, the parametric resonance which takes place for the smaller scales than the Jeans scale can enhance the perturbation of the ULA before the matter-radiation equality. When $x_{\text {osc }}$ is not too large, after the matter-radiation equality, $k_{r}$ soon becomes smaller than $k_{J}$, which increases as $a^{1 / 4}$ in MD. Then, the density perturbation of the ULA with $k_{r}$ starts to grow due to the Jeans instability. Therefore, a mild enhancement also can supply the missing small scale structures, asserted in Ref. [20]. In fact, in Ref. [25], it was argued that a mild enhancement around $k_{J}$ can lead to a significant enhancement of the low-mass halo abundance even for the conventional cosine potential by accepting a careful tuning of the initial condition $[26,27]$. By contrast, in the plateau case, we can evade the fine-tuning issue.

\section{GWs emission and Oscillon formation}

When the resonance instability continues, the linear perturbation ceases to be a good approximation, even if we start with an almost homogeneous initial condition. In the subsequent and transient stage, the oscillating axion in a highly inhomogeneous spatial configuration leads to a prominent emission of the gravitational waves (GWs) [13,14,28]. In contrast to the GWs emitted during the reheating, the peak frequency of the GW spectrum emitted later times can be in sensitivity bands of GW detectors. Here, we roughly evaluate the peak frequency of the GW emitted either during RD or MD (the later MD) as $f_{0} \simeq m /\left(1+z_{*}\right)$, where $z_{*}$ denotes the redshift at the emission. In the following, for simplicity, we identify the Hubble parameter at the emission as the one at the onset of the oscillation, i.e., $H\left(z_{*}\right) \simeq m / x_{\text {osc }}$, assuming that the $\mathrm{GW}$ emission takes place immediately after $x=x_{o s c}$ in the cosmological time scale. Then, the frequency of the GWs emitted during RD can be given by

$f_{0} \simeq\left(\frac{m}{10^{4} \mathrm{eV}} x_{\text {osc }}\right)^{\frac{1}{2}} \mathrm{~Hz} \quad(\mathrm{RD})$, 
where we used $\left(1+z_{*}\right) \simeq\left(H\left(z_{*}\right) / H_{e q}\right)^{1 / 2}\left(1+z_{e q}\right)$. Similarly, using $\left(1+z_{*}\right) \simeq\left(H\left(z_{*}\right) / H_{e q}\right)^{2 / 3}\left(1+z_{e q}\right)$, we obtain the frequency of the GWs emitted during MD as

$$
f_{0} \simeq\left(\frac{m}{10^{20} \mathrm{eV}} x_{o s c}^{2}\right)^{\frac{1}{3}} \mathrm{~Hz} \quad(\mathrm{MD})
$$

When we avoid choosing a hierarchically large value of $\left|\tilde{\phi}_{i}\right|$, $x_{o s c}$ ranges in $O(1) \lesseqgtr x_{o s c} \lesseqgtr O\left(10^{4}\right)$. For a direct detection, the GWs emitted during $\mathrm{RD}$ is more promising, e.g., for $m \simeq 10^{-6} \mathrm{eV}$ and $x_{o s c} \simeq 10^{4}$, the frequency $f_{0}$ is in the band of space interferometers $[29,30]$ and for $m \simeq 10^{3} \mathrm{eV}$ and $x_{\text {osc }} \simeq 10^{4}, f_{0}$ is in the band of ground based interferometers [31-33]. (When axions have unsuppressed interactions with the electromagnetic field, the larger mass range should be excluded because of the photon decay process $[1,18,19]$.) Meanwhile, for $m \simeq 10^{-16} \mathrm{eV}, f_{0}$ is in the detectable range by pulsar timing arrays [34-36].

In order to compute the amplitude of GWs, we introduce a parameter $\epsilon(\leq 1)$ which denotes the ratio between the relative spectral energy density of the emitted GWs and that of the homogeneous axion at the onset of the oscillation, which is of $O\left((m f)^{2}\right)$. Assuming that GWs were emitted just after the onset of the oscillation (in cosmological time scales), we obtain $\Omega_{g w}$ as [37]

$$
\Omega_{g w} \simeq \epsilon \Omega_{r} \frac{(m f)^{2}}{\rho_{e q}}\left(\frac{a_{o s c}}{a_{e q}}\right)^{4} \simeq 10^{-8} \epsilon x_{o s c}^{2}\left(\frac{f}{10^{16} \mathrm{GeV}}\right)^{2}
$$

In particular, for $m \gtrsim 10^{-27} \mathrm{eV}$, the axion starts to oscillate before the equal time, behaving as a dark matter component. Then, using Eq.(4), we obtain

$\Omega_{g w} \simeq 10^{-4} \epsilon \beta_{\phi} \sqrt{\frac{x_{o s c}}{10^{4}}} \sqrt{\frac{10^{-22} \mathrm{eV}}{m}}$.

When the axion is the dominant component of dark matter, i.e., $\beta_{\phi} \simeq 1$ and $x_{\text {osc }}=O\left(10^{4}\right)$, we obtain $\Omega_{g w} \simeq 10^{-7} \epsilon$ for $m \simeq 10^{-16} \mathrm{eV}$. Meanwhile, we obtain $\Omega_{g w} \simeq 10^{-12} \epsilon$ for $m \simeq 10^{-6} \mathrm{eV}$ and $\Omega_{g w} \simeq 10^{-16} \epsilon$ for $m \simeq 10^{3} \mathrm{eV}$. Therefore, for $\epsilon \gtrsim 10^{-6}$, we can expect a detection of GWs emitted by the resonantly oscillating modes of the string axions, using the pulsar timing arrays.

In Ref. [24], to evaluate the GW amplitude accurately, we have conducted the lattice simulation. Our analysis indicates that axions which were initially located at plateau regions potentially lead to a detectable emission of GWs, opening a new window of string axiverse.

Meanwhile, in Refs. [8-12], it was numerically shown that the long-lasting instability can lead to a fragmented configuration of the oscillating scalar field. Since the cosmic expansion is not crucial at the oscillon formation, clumps of the oscillon can be formed also during RD and the later MD. This will be also shown in Ref. [24].

\section{Summary: new window in string axiverse}

In this paper, we initiated a study on phenomenological imprints of string axions which were located at plateau regions before they commence to oscillate. We found that for such axions, the resonance instability can last without the disturbance of the cosmic expansion, because the delayed onset of the oscillation makes the redshift of the resonant modes insignificant. This instability takes place slightly below the Jeans scale, suggesting various implications on the structure formation of ULA dark matter. The persistent resonance instability leads to the emission of the detectable GWs and the subsequent oscillon formation [24]. In contrast to the GWs emitted during the reheating, the GWs at later times, discussed in this paper, can be emitted in the directly detectable ranges.

Acknowledgements We would like to thank A. Aoki and H. Tashiro for fruitful discussions and especially N. Kitajima for the collaboration in our follow-up study in [24]. Y.U. also thanks the organizers and participants of the workshop "Post-inflationary string Cosmology," during which this work was completed. This work was in part supported by JSPS KAKENHI Grant numbers 17H02894, 17K18778 (J.S.), 16K17689 (Y.U.), and MEXT KAKENHI Grant Numbers 15H05895, 17 H06359 (J.S.), 16H01095(Y.U.). Y. U. is also supported by Daiko foundation.

Open Access This article is distributed under the terms of the Creative Commons Attribution 4.0 International License (http://creativecomm ons.org/licenses/by/4.0/), which permits unrestricted use, distribution, and reproduction in any medium, provided you give appropriate credit to the original author(s) and the source, provide a link to the Creative Commons license, and indicate if changes were made.

Funded by SCOAP ${ }^{3}$.

\section{References}

1. A. Arvanitaki, S. Dimopoulos, S. Dubovsky, N. Kaloper, J. MarchRussell, Phys. Rev. D 81, 123530 (2010)

2. S. Dubovsky, A. Lawrence, M.M. Roberts, JHEP 1202, 053 (2012)

3. Y. Nomura, T. Watari, M. Yamazaki. arXiv:1706.08522 [hep-ph]

4. J.H. Traschen, R.H. Brandenberger, Phys. Rev. D 42, 2491 (1990). https://doi.org/10.1103/PhysRevD.42.2491

5. A.D. Dolgov, D.P. Kirilova, Sov. J. Nucl. Phys. 51, 172 (1990)

6. A.D. Dolgov, D.P. Kirilova, Yad. Fiz. 51, 273 (1990)

7. L. Kofman, A.D. Linde, A.A. Starobinsky, Phys. Rev. D 56, 3258 (1997). https://doi.org/10.1103/PhysRevD.56.3258 [hep$\mathrm{ph} / 9704452]$

8. S. Kasuya, M. Kawasaki, F. Takahashi, Phys. Lett. B 559, 99 (2003)

9. M.A. Amin, D. Shirokoff, Phys. Rev. D 81, 085045 (2010)

10. M.A. Amin, R. Easther, H. Finkel, R. Flauger, M.P. Hertzberg, Phys. Rev. Lett. 108, 241302 (2012)

11. M.A. Amin, M.P. Hertzberg, D.I. Kaiser, J. Karouby, Int. J. Mod. Phys. D 24, 1530003 (2014)

12. K. D. Lozanov, M. A. Amin. arXiv:1710.06851 [astro-ph.CO]

13. S. Antusch, F. Cefala, S. Orani, Phys. Rev. Lett. 118(1), 011303 (2017)

14. S. Antusch, F. Cefala, S. Krippendorf, F. Muia, S. Orani, F. Quevedo. arXiv:1708.08922 [hep-th]

15. D. Yoshida and J. Soda. arXiv:1708.09592 [gr-qc] 
16. J.P. Conlon, F. Quevedo, K. Suruliz, JHEP 0508, 007 (2005)

17. J. Halverson, C. Long, P. Nath. arXiv:1703.07779 [hep-ph]

18. D.J.E. Marsh, Phys. Rept. 643, 1 (2016)

19. L. Hui, J.P. Ostriker, S. Tremaine, E. Witten, Phys. Rev. D 95(4), 043541 (2017)

20. V. Irsic, M. Viel, M.G. Haehnelt, J.S. Bolton, G.D. Becker, Phys. Rev. Lett. 119(3), 031302 (2017)

21. R. Kallosh, A. Linde, JCAP 1307, 002 (2013)

22. R. Kallosh, A. Linde, D. Roest, JHEP 1311, 198 (2013)

23. R. Kallosh, A. Linde, D. Roest, Phys. Rev. Lett. 112(1), 011303 (2014)

24. N. Kitajima, J. Soda, Y. Urakawa. arXiv:1807.07037 [astro-ph.CO]

25. H.Y. Schive, T. Chiueh. arXiv:1706.03723 [astro-ph.CO]

26. U.H. Zhang, T. Chiueh, Phys. Rev. D 96(2), 023507 (2017)

27. U.H. Zhang, T. Chiueh, Phys. Rev. D 96(6), 063522 (2017)

28. S.Y. Zhou, E.J. Copeland, R. Easther, H. Finkel, Z.G. Mou, P.M. Saffin, JHEP 1310, 026 (2013)
29. P. Amaro-Seoane et al., GW Notes 6, 4 (2013). N. Seto, S. Kawamura and T. Nakamura. Phys. Rev. Lett. 87, 221103 (2001)

30. N. Seto, S. Kawamura, T. Nakamura, Phys. Rev. Lett. 87, 221103 (2001)

31. J. Aasi et al., LIGO Scientific Collaboration, Class. Quant. Grav. 32, 074001 (2015)

32. F. Acernese et al., VIRGO Collaboration, Class. Quant. Grav. 32(2), $024001(2015)$

33. K. Somiya, KAGRA Collaboration, Class. Quant. Grav. 29, 124007 (2012)

34. S.L. Detweiler, Astrophys. J. 234, 1100 (1979)

35. L. Lentati et al., Mon. Not. R. Astron. Soc. 453(3), 2576 (2015)

36. Z. Arzoumanian et al., NANOGrav Collaboration, Astrophys. J. 821(1), 13 (2016)

37. S.Y. Khlebnikov, I.I. Tkachev, Phys. Rev. D 56, 653 (1997) 